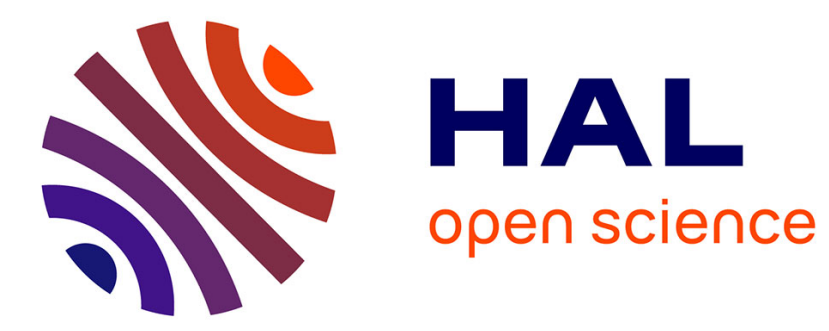

\title{
Approximate controllability and stabilizability of a linearized system for the interaction between a viscoelastic fluid and a rigid body
}

\author{
Debanjana Mitra, Arnab Roy, Takéo Takahashi
}

\section{- To cite this version:}

Debanjana Mitra, Arnab Roy, Takéo Takahashi. Approximate controllability and stabilizability of a linearized system for the interaction between a viscoelastic fluid and a rigid body. Mathematics of Control, Signals, and Systems, 2021. hal-02987407

\section{HAL Id: hal-02987407 \\ https://hal.science/hal-02987407}

Submitted on 3 Nov 2020

HAL is a multi-disciplinary open access archive for the deposit and dissemination of scientific research documents, whether they are published or not. The documents may come from teaching and research institutions in France or abroad, or from public or private research centers.
L'archive ouverte pluridisciplinaire HAL, est destinée au dépôt et à la diffusion de documents scientifiques de niveau recherche, publiés ou non, émanant des établissements d'enseignement et de recherche français ou étrangers, des laboratoires publics ou privés. 


\title{
Approximate controllability and stabilizability of a linearized system for the interaction between a viscoelastic fluid and a rigid body
}

\author{
Debanjana Mitra ${ }^{1}$, Arnab Roy ${ }^{2}$, and Takéo Takahashi ${ }^{3}$ \\ ${ }^{1}$ Department of Mathematics, Indian Institute of Technology Bombay, Powai, Maharashtra \\ 400076, India \\ ${ }^{2}$ Institute of Mathematics, Czech Academy of Sciences, Žitná 25, 11567 Praha 1, Czech \\ Republic \\ ${ }^{3}$ Université de Lorraine, CNRS, Inria, IECL, F-54000 Nancy, France (takeo.takahashi@inria.fr)
}

October 21, 2020

\begin{abstract}
We study control properties of a linearized fluid-structure interaction system, where the structure is a rigid body and where the fluid is a viscoelastic material. We establish the approximate controllability and the exponential stabilizability for the velocities of the fluid and of the rigid body and for the position of the rigid body. In order to prove this, we prove a general result for this kind of systems that generalizes in particular the case without structure. The exponential stabilization of the system is obtained with a finite-dimensional feedback control acting only on the momentum equation on a subset of the fluid domain and up to some rate that depends on the coefficients of the system. We also show that, as in the case without structure, the system is not exactly null-controllable in finite time.
\end{abstract}

Keywords. Fluid-structure interaction systems, viscoelastic fluids, controllability, stabilizability, finite dimensional controls.

2010 Mathematics Subject Classification. 76A10, 74F10, 93B52, 93D15, 35Q35.

\section{Contents}

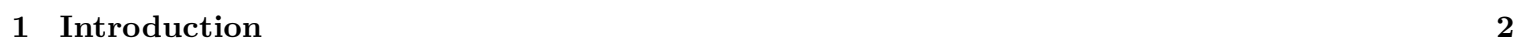

\begin{tabular}{|lr}
\hline 2 & An abstract result
\end{tabular}

\begin{tabular}{|lr|}
\hline 3 Case of multiple modes & 11 \\
\hline
\end{tabular}

\begin{tabular}{|lr|r}
\hline & Proof of Theorem 1.1 & 14
\end{tabular}

\begin{tabular}{|lr|}
\hline 5 & Lack of null controllability \\
\hline
\end{tabular}

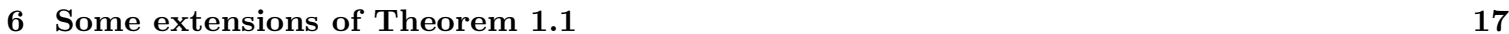

\section{Introduction}

We study some controllability properties of a mathematical model describing the motion of a rigid body immersed in a viscoelastic fluid. First we describe the corresponding model: we denote by $\Omega \subset \mathbb{R}^{3}$ a 
bounded domain containing the fluid and the rigid body. The structure domain $\mathcal{S}(t)$ can be described through its orientation $Q(t) \in S O(3)$ and its center of mass $h(t) \in \mathbb{R}^{3}$, whereas the fluid domain $\mathcal{F}(t)$ is the complement of the structure domain in $\Omega$ :

$$
\mathcal{S}(t)=h(t)+Q(t) \mathcal{S}_{0}, \quad \mathcal{F}(t)=\Omega \backslash \overline{\mathcal{S}(t)},
$$

where $\mathcal{S}_{0} \subset \mathbb{R}^{3}$ is the reference domain of the rigid body, chosen as a nonempty regular domain with center of mass 0 .

Now, to describe the dynamics of the corresponding fluid-structure interaction system, we use the Newton laws to obtain the equations below on the linear and angular velocities of the rigid body $\ell \in \mathbb{R}^{3}$ and $\omega \in \mathbb{R}^{3}$ :

$$
\begin{gathered}
\left\{\begin{array}{c}
h^{\prime}=\ell \quad \forall t>0, \\
Q^{\prime}=\mathbb{S}(\omega) Q \quad \forall t>0,
\end{array}\right. \\
m \ell^{\prime}=-\int_{\partial \mathcal{S}(t)} \tau_{\text {fluid }} n d \Gamma \quad t>0, \\
(J \omega)^{\prime}=-\int_{\partial \mathcal{S}(t)}(x-h) \times \tau_{\text {fluid }} n d \Gamma \quad t>0,
\end{gathered}
$$

where $\tau_{\text {fluid }}$ is the stress tensor of the fluid, where $m>0$ and $J$ are the mass and the moment of inertia of the rigid body and where

$$
\mathbb{S}(\omega)=\left[\begin{array}{ccc}
0 & -\omega_{3} & \omega_{2} \\
\omega_{3} & 0 & -\omega_{1} \\
-\omega_{2} & \omega_{1} & 0
\end{array}\right] \quad\left(\omega \in \mathbb{R}^{3}\right)
$$

For the fluid, its velocity $u$ and pressure $p$ satisfy

$$
\begin{gathered}
\left\{\begin{array}{c}
\frac{\partial u}{\partial t}+(u \cdot \nabla) u-\operatorname{div} \tau_{\text {fluid }}=f \chi_{\mathcal{O}} \quad t>0, x \in \mathcal{F}(t), \\
\operatorname{div} u=0 \quad t>0, x \in \mathcal{F}(t),
\end{array}\right. \\
\left\{\begin{array}{c}
u=0 \quad t>0, x \in \partial \Omega, \\
u(t, x)=\ell(t)+\omega(t) \times(x-h(t)) \quad t>0, x \in \partial \mathcal{S}(t),
\end{array}\right.
\end{gathered}
$$

where $f$ is the control of the system, acting on a part of the fluid domain $\mathcal{O} \subset \Omega$ ( $\chi_{\mathcal{O}}$ is the characteristic function of $\mathcal{O})$. We assume that $\mathcal{O}$ is a nonempty domain with $\mathcal{O} \subset \mathcal{F}(t)$ for all $t$.

Finally, it remains to describe the stress tensor of the fluid. For that, we consider the Johnson-Segalman model for viscoelastic flows, that is

$$
\tau_{\text {fluid }}=\Sigma(u, p)+\tau,
$$

where

and where

$$
\Sigma(u, p)=2 \eta \mathbb{D}(u)-p \mathbb{I}_{3}, \quad \mathbb{D}(u)=\frac{1}{2}\left(\nabla u+\nabla u^{\top}\right)
$$

$$
\frac{\partial \tau}{\partial t}+(v \cdot \nabla) \tau+g_{a}(\tau, \nabla u)+\lambda \tau=2 \kappa \mathbb{D} u \quad t>0, x \in \mathcal{F}(t)
$$

Here $a \in[-1,1]$ is a constant and

$$
g_{a}(\tau, \nabla u)=\tau \mathbb{W}(u)-\mathbb{W}(u) \tau-a(\mathbb{D}(u) \tau+\tau \mathbb{D}(u)), \quad \mathbb{W}(u)=\frac{1}{2}\left(\nabla u-\nabla u^{\top}\right) .
$$

In the above relations, $\eta, \lambda$ and $\kappa$ are positive constants and $\mathbb{W}(u)$ denotes the vorticity tensor. Local existence of solutions of viscoelastic fluids for arbitrary data and global existence of solutions for sufficiently small data in appropriate spaces have been established in [14, 12. Regarding a detailed discussion on Johnson-Segalman fluid flow and viscoelastic fluids in general, we refer to [26].

The corresponding system (1.1), 1.2, 1.3, 1.4, 1.5 with initial conditions for $u, \tau, h, Q, \ell$ and $\omega$ is already studied in 13 and 32 . They show the well-posedness of the system respectively in a $L^{p}-L^{q}$ setting and in Hilbert setting. Their methods are based on a change of variables to handle the moving and unknown fluid domain $\mathcal{F}(t)$, a linearization and a fixed point argument. Due to the viscoelastic part corresponding to $\tau$, the system is more complex to study than the system of interaction between a rigid body and Newtonian 
fluid governed by the classical Navier-Stokes system (that is $\tau=0$ in the above system). In particular in the linearization, the equation of $\tau$ is treated separately.

Here our aim is to present a linearization of the above system and to study some control properties of this linearization, seen as a first step towards some control results for the nonlinear system. A similar linearization is used for the well-posedness and the controllability results of the system composed by a rigid body and Newtonian fluid (see for instance, 15, 4, 3]). The idea is to consider a change of variables to write the above system with fixed domains $\mathcal{F}$ and $\mathcal{S}$ and then remove all the nonlinear terms coming from the model and from the change of variables. This leads to the following system

$$
\begin{aligned}
& \left\{\begin{array}{c}
\frac{\partial u}{\partial t}-\operatorname{div}(\Sigma(u, p)+\tau)=f \chi_{\mathcal{O}} \quad t>0, y \in \mathcal{F}, \\
\operatorname{div} u=0 \quad t>0, y \in \mathcal{F}, \\
\frac{\partial \tau}{\partial t}+\lambda \tau=2 \kappa \mathbb{D} u \quad t>0, y \in \mathcal{F},
\end{array}\right. \\
& \left\{\begin{array}{c}
u=0 \quad t>0, y \in \partial \Omega, \\
u(t, y)=\ell(t)+\omega(t) \times y \quad t>0, y \in \partial \mathcal{S},
\end{array}\right. \\
& \left\{\begin{array}{cl}
m \ell^{\prime} & =-\int_{\partial \mathcal{S}}(\Sigma(u, p)+\tau) n d \Gamma \quad t>0, \\
J_{0} \omega^{\prime}=-\int_{\partial \mathcal{S}} y \times(\Sigma(u, p)+\tau) n d \Gamma \quad t>0, &
\end{array}\right. \\
& \left\{\begin{array}{cc}
h^{\prime}=\ell & \forall t>0 \\
\theta^{\prime}=\omega & \forall t>0 .
\end{array}\right.
\end{aligned}
$$

Note that in the above system, $\mathcal{F}$ and $\mathcal{S}$ are now time-independent regular domains with $\mathcal{F}=\Omega \backslash \overline{\mathcal{S}}$. We keep the same notation as in (1.1), 1.2, $1.3,1.4,1.5$ for $u, \tau, \ell, \omega$ but these variables have been modified through the change of variables. $J_{0}$ is time-independent positive symmetric matrix and the rotation matrix $Q$ has been replaced by a local chart of $S O(3)$ (for instance the Euler angles), that is $\theta(t) \in \mathbb{R}^{3}$. If we introduce the density $\rho_{\mathcal{S}}$ of the rigid body, and we assume it is a positive constant, we have the following relation:

$$
m=\rho_{\mathcal{S}}|\mathcal{S}|, \quad J_{0}=\rho_{\mathcal{S}} \int_{\mathcal{S}}\left(|y|^{2} \mathbb{I}_{3}-y \otimes y\right) d y
$$

where $|\mathcal{S}|$ is the Lebesgue measure of $\mathcal{S}$. We can assume that 0 is the center of gravity of $\mathcal{S}$ so that

$$
\int_{\mathcal{S}} y d y=0
$$

In what follows, we only consider a particular case of $(1.6)-(1.9)$, where we assume that $\tau$ is of the form $\tau=2 \mathbb{D}(v)$. Due to the incompressibility condition of $u$ and its boundary values, we finally consider the following linear system:

$$
\begin{aligned}
& \left\{\begin{aligned}
\frac{\partial u}{\partial t}-\operatorname{div}(\Sigma(u, p)+2 \mathbb{D}(v)) & =f \chi_{\mathcal{O}} \quad t>0, y \in \mathcal{F}, \\
\operatorname{div} u & =0 \quad t>0, y \in \mathcal{F}
\end{aligned}\right. \\
& \left\{\begin{array}{c}
u=0 \quad t>0, y \in \partial \Omega \\
u(t, y)=\ell(t)+\omega(t) \times y \quad t>0, y \in \partial \mathcal{S}
\end{array}\right. \\
& \left\{\begin{array}{c}
m \ell^{\prime}=-\int_{\partial \mathcal{S}}(\Sigma(u, p)+2 \mathbb{D}(v)) n d \Gamma \quad t>0, \\
J_{0} \omega^{\prime}=-\int_{\partial \mathcal{S}} y \times(\Sigma(u, p)+2 \mathbb{D}(v)) n d \Gamma \quad t>0,
\end{array}\right. \\
& \left\{\begin{array}{c}
\frac{\partial v}{\partial t}+\lambda v=\kappa u \quad t>0, y \in \mathcal{F} \\
\operatorname{div} v=0 \quad t>0, y \in \mathcal{F}
\end{array}\right. \\
& \left\{\begin{array}{c}
v=0 \quad t>0, y \in \partial \Omega, \\
v(t, y)=k(t)+r(t) \times y \quad t>0, y \in \partial \mathcal{S},
\end{array}\right.
\end{aligned}
$$




$$
\begin{aligned}
& \begin{cases}k^{\prime}+\lambda k=\kappa \ell & \forall t>0 \\
r^{\prime}+\lambda r=\kappa \omega & \forall t>0\end{cases} \\
& \begin{cases}h^{\prime}=\ell & \forall t>0 \\
\theta^{\prime}=\omega & \forall t>0\end{cases} \\
& \left\{\begin{array}{c}
u(0, \cdot)=u^{0}, \quad v(0, \cdot)=v^{0} \text { in } \mathcal{F}, \\
h(0)=h^{0}, \quad \theta(0)=\theta^{0}, \quad \ell(0)=\ell^{0}, \quad \omega(0)=\omega^{0}, \quad k(0)=k^{0}, \quad r(0)=r^{0} .
\end{array}\right.
\end{aligned}
$$

In order to study the above system, it is standard to extend $u$ and $v$ as functions of $\Omega$ by setting

$$
u(t, y)=\ell(t)+\omega(t) \times y, \quad v(t, y)=k(t)+r(t) \times y \quad(t \geqslant 0, y \in \mathcal{S})
$$

and similar formula for $u^{0}$ and $v^{0}$. This leads us to introduce the following space

$$
\mathbb{H}=\left\{u \in L^{2}(\Omega) ; \operatorname{div} u=0 \text { in } \Omega, \mathbb{D}(u)=0 \text { in } \mathcal{S}, u \cdot n=0 \text { on } \partial \Omega\right\} .
$$

We recall (see [36. Lemma 1.1, p.18]) that $\mathbb{D}(u)=0$ in $\mathcal{S}$, if and only if there exist $\ell_{u}, \omega_{u} \in \mathbb{R}^{3}$ such that

$$
u(y)=\ell_{u}+\omega_{u} \times y \quad(y \in \mathcal{S}) .
$$

We consider the inner product on $L^{2}(\Omega)$ defined by

$$
(u, v)=\int_{\mathcal{F}} u \cdot v d y+\int_{\mathcal{S}} \rho_{\mathcal{S}} u \cdot v d y
$$

where $\rho_{\mathcal{S}}$ is the density of the rigid body. The corresponding norm is equivalent to the usual norm in $L^{2}(\Omega)$ and if $u, v \in \mathbb{H}$, then we have:

$$
(u, v)=\int_{\mathcal{F}} u \cdot v d y+m \ell_{u} \cdot \ell_{v}+J_{0} \omega_{u} \cdot \omega_{v} .
$$

We also define the spaces

$$
\mathbb{H}_{1 / 2}=\left\{u \in H_{0}^{1}(\Omega) ; \operatorname{div} u=0 \text { in } \Omega, \mathbb{D}(u)=0 \text { in } \mathcal{S}\right\}, \quad \text { and } \quad \mathbb{H}_{1}=\left\{u \in \mathbb{H}_{1 / 2} ; u_{\mid \mathcal{F}} \in H^{2}(\mathcal{F})\right\} .
$$

Then, we will show that if $u^{0} \in \mathbb{H}_{1 / 2}, v^{0} \in \mathbb{H}_{1},\left(h^{0}, \theta^{0}\right) \in \mathbb{R}^{6}$ and $f \in L^{2}\left(0, T ; L^{2}(\mathcal{O})\right)$, there exists a unique solution to $1.12-1.19$

$$
u \in H^{1}(0, T ; \mathbb{H}) \cap L^{2}\left(0, T ; \mathbb{H}_{1}\right) \cap C^{0}\left([0, T] ; \mathbb{H}_{1 / 2}\right), \quad v \in H^{1}\left(0, T ; \mathbb{H}_{1}\right), \quad(h, \theta) \in H^{1}\left(0, T ; \mathbb{R}^{6}\right) .
$$

Note that it implies in particular that

$$
(\ell, \omega, k, r) \in H^{1}\left(0, T ; \mathbb{R}^{12}\right) .
$$

We say that $1.12-1.19$ is approximately controllable in time $T>0$ if for any $u^{0}, u^{1} \in \mathbb{H}_{1 / 2}, v^{0}, v^{1} \in \mathbb{H}_{1}$, $\left(h^{0}, \theta^{0}\right),\left(h^{1}, \theta^{1}\right) \in \mathbb{R}^{6}$ and for any $\varepsilon>0$, there exists $f \in L^{2}\left(0, T ; L^{2}(\mathcal{O})\right)$ such that the solution to 1.12 1.19 satisfies

$$
\|[u(T, \cdot), v(T, \cdot), h(T), \theta(T)]-\left[u^{1}, v^{1}, h^{1}, \theta^{1} \|_{\mathbb{H}_{1 / 2} \times \mathbb{H}_{1} \times \mathbb{R}^{6}}<\varepsilon .\right.
$$

In the earlier works on controllability of linear viscoelastic models [19, 18, 17, Leugering considered an integro-differential equation and focus on the control of the velocity but not on the residual stresses. Then, in 9], the authors addressed the problem of control for both the velocity and the stress of a viscoelastic material. Precisely, they stated an approximate controllability result for fluids of the Jeffreys kind (system $(1.6)$ ) and null approximate controllability results for fluids of the Maxwell kind (that is system 1.6 with $\eta=0$ in $\Sigma(u, p)$ ). In [27, Renardy considered one-dimensional shear flows of multimode linear Maxwell and Jeffreys fluids (see (6.6) with a distributed control. Exact controllability for single-mode Maxwell fluids, approximate controllability of multimode Maxwell and Jeffreys fluids hold when the control is restricted to a subinterval and exact controllability in the case of several relaxation modes holds if the control is on the entire interval. In [8], the authors considered higher-dimensional single mode Jeffreys fluid and established the approximate controllability result in an arbitrarily small time only for the velocity with distributed or boundary controls supported by arbitrarily small sets. The controllability properties for both the velocity and the stress of a 
single mode Maxwell model in higher dimension with distributed or boundary controls is analyzed in [2]: they proved the large time approximate controllability and under a geometric condition imposed on the controlled region along with an additional restriction on the constants $\lambda$ and $\kappa$, a large time exact controllability was also established. Several improvements of these results on controllability of [8, 2] have been done in [5] where the authors proved the approximate controllability of both the velocity and the stress for single and multi-mode Jeffreys fluids. Under the usual geometric condition on the controlled domain, they established the exact controllability with interior controls for the single mode and the approximate controllability for multimode Maxwell models. The lack of null controllability of Jeffreys and Maxwell fluids with distributed control is considered in 21. They established that the solution of single mode and multimode Jeffreys systems cannot reach zero. Due to the finite speed of propagation property, the single mode Maxwell system cannot be null controllable for small time whereas the multimode Maxwell system is not null controllable for any time.

In all these results for linear viscoelastic flows in higher dimensions, control of the stress tensor is possible only under the constraint that the stress is the symmetric part of a gradient. This is the main hindrance to work with nonlinear viscoelastic model and only few results are available 28, 29, 33. regarding the characterization of the set of reachable states.

Let us mention some works concerning the controllability of fluid-structure interaction system. Regarding one-dimensional viscous Burgers-particle system, Doubova and Fernández-Cara proved in [7 that the local null controllability holds by boundary controls acting on both ends of the finite interval. Later, Liu et. al. 20 improved this result by using only one control (located at one end of the interval). A simplified 2D model where the fluid equations are replaced by the Helmholtz equations and the structure is modeled by a harmonic oscillator is considered in [25] and the exact controllability is established with an internal control acting only in the fluid part. In the case of a $2 \mathrm{D}$ fluid-structure system where a rigid ball is moving inside a viscous, incompressible Navier-Stokes fluid, the exact controllability with an internal control in the fluid equation is proved in [16. In 4, Boulakia and Osses obtained the same result but for a body of more general shape. These results have been extended to 3D and for a general shaped rigid body in [3]. Finally, the authors in 30 prove the local null controllability for a 2D Boussinesq flow in interaction with a rigid body by using controls acting only on the temperature equation.

The above mentioned works related to fluid-structure interaction systems correspond to the case where the control acts on the fluid. Some articles are available concerning the case where the control is supported on the structure. In [6], the authors deal with the one-dimensional case and the null controllability for the velocities of the fluid and of the particle and the approximate controllability for the position of the particle are established with a control acting only on the particle. Note that in this result, no smallness assumption is considered, but the time of controllability can be large and may depends on the initial data and the final data. In 23], the authors show that this time of controllability can be uniform with respect to the initial data. In 24], the structure is a deformable beam located at the boundary of the fluid domain and the author obtains the local stabilization of the corresponding system. In 35] (respectively 31), an open stabilization result is proved in the case of rigid ball moving into viscous incompressible (respectively compressible) fluid with a spring-damper type control.

In the literature, there are no available controllability or stabilizability results concerning the motion of a rigid body inside a viscoelastic fluid. In this article, we want to explore the control properties for fluidstructure system (1.12)-(1.19). This is the first result in the context of controllability and stabilizability of viscoelastic fluid-rigid body interaction problem. More precisely, our main result is the following one

Theorem 1.1. Assume $\mathcal{O}$ is a nonempty open subset of $\mathcal{F}$. Then the linear system $1.12-1.19$ is approximately controllable. Assume moreover that

$$
0<\beta<\lambda+\frac{\kappa}{\eta}
$$

Then the system 1.12-1.19 is exponentially stabilizable with rate $-\beta$ and with a feedback of finite dimension: there exists

$$
\left[\phi^{j}, \psi^{j}, \alpha^{j}\right] \in \mathbb{H} \times \mathbb{H}_{1} \times \mathbb{R}^{6}, \quad w^{j} \in L^{2}(\mathcal{O}) \quad(j=1, \ldots, K), \quad C>0
$$

such that the system $1.12-1.19$ with

$$
f=\sum_{j=1}^{K}\left(\left\langle\phi^{j}, u\right\rangle_{\mathbb{H}}+\left\langle\psi^{j}, v\right\rangle_{\mathbb{H}_{1}}+\left\langle\alpha^{j},(h, \theta)\right\rangle_{\mathbb{R}^{6}}\right) w^{j}
$$


admits a unique solution $[u, v, h, \theta] \in C^{0}\left([0, \infty) ; \mathbb{H} \times \mathbb{H}_{1} \times \mathbb{R}^{6}\right)$, with

$$
\|u(t, \cdot)\|_{\mathbb{H}}+\|v(t, \cdot)\|_{\mathbb{H}_{1}}+\|(h(t), \theta(t))\|_{\mathbb{R}^{6}} \leqslant C e^{-\beta t}\left(\left\|u^{0}\right\|_{\mathbb{H}}+\left\|v^{0}\right\|_{\mathbb{H}_{1}}+\left\|\left(h^{0}, \theta^{0}\right)\right\|_{\mathbb{R}^{6}}\right) \quad(t \geqslant 0) .
$$

This result will be a consequence of a general result on approximate controllability that we state and prove in Section 2. We start with an abstract operator form and we analyze spectral properties of the corresponding operators. The approximate controllability and the exponential stabilizability of the abstract system follow from the well-known Fattorini criterion (see, for instance [1]). We extend this abstract result from single mode to the multiple modes framework in Section 3 . The analysis concerning behavior of eigenvalues and generalized eigenfunctions is more technical in this multimode part. Section 4 is devoted to the proof of the main result of the paper. The idea is to define appropriate operators and spaces corresponding to the fluid-structure interaction system so that we can apply our abstract result obtained in Section 2 Precisely, the Fattorini criterion reduces to a unique continuation problem for fluid-structure interaction system that we deal in this section. In Section 5 , we show that if the support of the control is not $\mathcal{F}$, then there exists an initial data such that for any control, the solution of the fluid-structure interaction system cannot be brought to a smooth trajectory in finite time and hence that the system is not null controllable. Finally, we take advantage of our abstract result in Section 2 to mention some possible extensions of the main result in Section 6 for instance the case of controls with a null component and the case of a fluid-rigid body system when the fluid follows a linear Jeffreys model with several relaxation mode.

The main novelties that we bring in this article are :

- In the literature, only existence results in appropriate spaces for the fluid-structure interaction system where a rigid body is moving inside a viscoelastic fluid are available (see [13, 32]). At the best of our knowledge, the controllability and stabilizability properties of such system have not yet been studied in the literature.

- We prove some general results in abstract framework on approximate controllability and stabilizability of coupled systems. As an application of that abstract result, the approximate controllability and exponential stabilizability results for the interaction between a single mode Jeffreys fluid (linearized version of nonlinear viscoelastic model) and a rigid body are established. Note that our abstract results permit to recover previous results in the case of a fluid without structure.

- Further, we show that the system is not exactly null controllable and hence the approximate controllability and the exponential stabilizability are the best possible results for the system in this direction.

- We have also extended our results to the interaction between Jeffreys fluid with multiple relaxation modes and a rigid body and the case of controls with a null-component.

\section{An abstract result}

In this section, we state and prove a general result that will imply Theorem 1.1 .

Let us consider $\mathbb{H}, \mathbb{U}$ Hilbert spaces, $A_{0}: \mathcal{D}\left(A_{0}\right) \rightarrow \mathbb{H}$ a self-adjoint positive operator with compact resolvent. We use the following notation:

$$
\mathbb{H}_{\gamma}:= \begin{cases}\mathcal{D}\left(A_{0}^{\gamma}\right) & \text { if } \gamma \geqslant 0 \\ \mathcal{D}\left(A_{0}^{-\gamma}\right)^{\prime} & \text { if } \gamma<0\end{cases}
$$

where $V^{\prime}$ stands for the dual space of $V$ with respect to the pivot space $\mathbb{H}$.

We also assume $B_{0} \in \mathcal{L}(\mathbb{U}, \mathbb{H})$ and $C_{0} \in \mathcal{L}\left(\mathbb{H}, \mathbb{R}^{N}\right)$, where $N \in \mathbb{N}^{*}$. From standard results on parabolic equations, we deduce from the above hypotheses that if $f \in L^{2}(0, T ; \mathbb{U})$ and $u^{0} \in \mathbb{H}_{1 / 2}$ then there exists a unique solution

of the Cauchy problem

$$
u \in H^{1}(0, T ; \mathbb{H}) \cap L^{2}\left(0, T ; \mathbb{H}_{1}\right) \cap C^{0}\left([0, T] ; \mathbb{H}_{1 / 2}\right)
$$

$$
\dot{u}+A_{0} u=B_{0} f, \quad t \in(0, T), \quad u(0)=u^{0} .
$$

Let us recall the definition of $\left(A_{0}, B_{0}\right)$ approximately controllable in time $T>0$ : for any $u^{0}, u^{1} \in \mathbb{H}_{1 / 2}$ and $\varepsilon>0$, there exists $f \in L^{2}(0, T ; \mathbb{U})$ such that the solution $u$ of 2.1 satisfies $\left\|u(T)-u^{1}\right\|_{\mathbb{H}_{1 / 2}}<\varepsilon$. Using the standard criterion of Fattorini-Hautus that we recall below, the time $T>0$ in the above definition can be chosen arbitrary. 
We consider $\eta, \lambda, \kappa \in \mathbb{R}_{+}^{*}$ and the following system

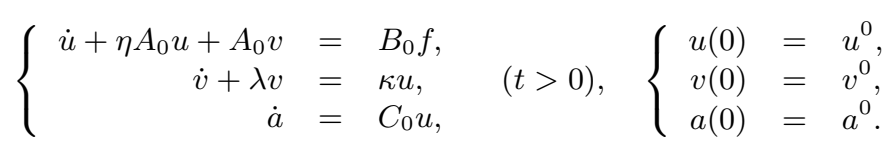

In the above system, $Z=[u, v, a]$ is the state and $f$ is the control. We will see in Section 4 that the system 1.12- 1.19 can be written under the above form.

We can write 2.2 as follows:

$$
\dot{Z}=A Z+B f, \quad t>0, \quad Z(0)=Z^{0},
$$

by defining

$$
\mathbb{X}:=\mathbb{H} \times \mathbb{H}_{1} \times \mathbb{R}^{N}, \quad \mathcal{D}(A):=\mathbb{H}_{1} \times \mathbb{H}_{1} \times \mathbb{R}^{N}, \quad A:=\left[\begin{array}{ccc}
-\eta A_{0} & -A_{0} & 0 \\
\kappa \mathrm{I} & -\lambda \mathrm{I} & 0 \\
C_{0} & 0 & 0
\end{array}\right], \quad B:=\left[\begin{array}{c}
B_{0} \\
0 \\
0
\end{array}\right],
$$

and

$$
Z=[u, v, a], \quad Z^{0}=\left[u^{0}, v^{0}, a^{0}\right] .
$$

First, we have the following result on $A$ :

Proposition 2.1. The operator $(A, \mathcal{D}(A))$, defined by 2.4 generates an analytic semigroup in $\mathbb{X}$. Its adjoint in $\mathbb{Y}:=\mathbb{H} \times \mathbb{H}_{-1} \times \mathbb{R}^{N}$ is given by

$$
\begin{gathered}
\mathcal{D}\left(A^{*}\right):=\left\{[\phi, \psi, \alpha] \in \mathbb{Y} ;-\eta A_{0} \phi+\kappa \psi \in \mathbb{H}\right\}, \\
A^{*}=\left[\begin{array}{ccc}
-\eta A_{0} & \kappa \mathrm{I} & C_{0}^{*} \\
-A_{0} & -\lambda \mathrm{I} & 0 \\
0 & 0 & 0
\end{array}\right] .
\end{gathered}
$$

Proof. First we split $A$ into two parts:

$$
A=A_{1}+A_{2}, \quad A_{1}:=\left[\begin{array}{ccc}
-\eta A_{0} & 0 & 0 \\
\kappa \mathrm{I} & 0 & 0 \\
0 & 0 & 0
\end{array}\right], \quad A_{2}:=\left[\begin{array}{ccc}
0 & -A_{0} & 0 \\
0 & -\lambda \mathrm{I} & 0 \\
C_{0} & 0 & 0
\end{array}\right]
$$

and we note that $A_{2} \in \mathcal{L}(\mathbb{X})$. Then, using that $A_{0}$ is self-adjoint and positive, we deduce that there exists $\vartheta \in(\pi / 2, \pi)$ such that for all $\mu \in \mathbb{C}^{*},|\arg \mu|<\vartheta$,

$$
\left\|\left(\mu \mathrm{I}-A_{1}\right)^{-1}\right\|_{\mathcal{L}(\mathbb{X})} \leqslant \frac{C}{|\mu|}
$$

which yields that $A_{1}$ generates an analytic semigroup in $\mathbb{X}$. We then apply Theorem 2.1 in [22, p.80], to deduce that $A=A_{1}+A_{2}$ also generates an analytic semigroup in $\mathbb{X}$.

Formulas 2.5 and 2.6 are obtained by standard computation.

From the above result and standard results on parabolic equations, we deduce that if

$$
Z^{0}=\left[u^{0}, v^{0}, a^{0}\right] \in \mathcal{D}\left(A^{1 / 2}\right)=\mathbb{H}_{1 / 2} \times \mathbb{H}_{1} \times \mathbb{R}^{N}, \quad f \in L^{2}(0, T ; \mathbb{U}),
$$

then 2.2 admits a unique solution

$$
u \in H^{1}(0, T ; \mathbb{H}) \cap L^{2}\left(0, T ; \mathbb{H}_{1}\right) \cap C^{0}\left([0, T] ; \mathbb{H}_{1 / 2}\right), \quad v \in H^{1}\left(0, T ; \mathbb{H}_{1}\right), \quad a \in H^{1}\left(0, T ; \mathbb{R}^{N}\right) .
$$

We say that 2.2 is approximately controllable in time $T>0$ if for any

$$
u^{0}, u^{1} \in \mathbb{H}_{1 / 2}, \quad v^{0}, v^{1} \in \mathbb{H}_{1}, \quad a^{0}, a^{1} \in \mathbb{R}^{N},
$$

and $\varepsilon>0$, there exists $f \in L^{2}(0, T ; \mathbb{U})$ such that the solution $[u, v, a]$ of 2.2 satisfies

$$
\left\|u(T)-u^{1}\right\|_{\mathbb{H}_{1 / 2}}+\left\|v(T)-v^{1}\right\|_{\mathbb{H}_{1}}+\left\|a(T)-a^{1}\right\|_{\mathbb{R}^{N}}<\varepsilon .
$$


We are also interested by stabilization results: we say that the system 2.2 is stabilizable in $\mathbb{X}$ with a rate lower than $-\beta$ if there exists $F_{\beta} \in \mathcal{L}(\mathbb{X}, \mathbb{U})$ such that $A_{\beta}:=A+B F_{\beta}$ with domain $\mathcal{D}\left(A_{\beta}\right)=\mathcal{D}(A)$ is the infinitesimal generator of an analytic and exponentially stable semigroup on $\mathbb{X}$ of type lower than $-\beta$. In our main result, we obtain a feedback operator $F_{\beta}$ of finite rank $K$. In that case, there exists

$$
\left[\phi^{j}, \psi^{j}, \alpha^{j}\right] \in \mathbb{X}, \quad w^{j} \in \mathbb{U} \quad(j=1, \ldots, K), \quad C>0
$$

such that for any

$$
Z^{0}=\left[u^{0}, v^{0}, a^{0}\right] \in \mathbb{X}
$$

the system 2.2 with

$$
f=\sum_{j=1}^{K}\left\langle\left[\phi^{j}, \psi^{j}, \alpha^{j}\right],[u, v, a]\right\rangle_{\mathbb{X}} w^{j}
$$

admits a unique solution $[u, v, a] \in C^{0}([0, \infty) ; \mathbb{X})$, with

$$
\|[u(t), v(t), a(t)]\|_{\mathbb{X}} \leqslant C e^{-\beta t}\left\|\left[u^{0}, v^{0}, a^{0}\right]\right\|_{\mathbb{X}} \quad(t \geqslant 0) .
$$

Our main result in this section is the following theorem:

Theorem 2.2. Assume $\left(A_{0}, B_{0}\right)$ is approximately controllable and that $B_{0}^{*} A_{0}^{-1} C_{0}^{*}: \mathbb{R}^{N} \rightarrow \mathbb{U}$ is injective. Then the system 2.2 is approximately controllable for any $T>0$.

Assume

$$
0<\beta<\lambda+\frac{\kappa}{\eta}
$$

Then, the system 2.2 is stabilizable in $\mathbb{X}$ with a rate lower than $-\beta$. More precisely, by denoting

$$
K:=\max \{\operatorname{dim} \operatorname{ker}(\mu \mathrm{I}-A) ; \mu \in \sigma(A), \operatorname{Re}(\mu) \geqslant-\beta\}<\infty,
$$

there exists $F_{\beta} \in \mathcal{L}(\mathbb{X}, \mathbb{U})$ with $\operatorname{rank}\left(F_{\beta}\right)=K$ such that $A_{\beta}:=A+B F_{\beta}$ with domain $\mathcal{D}\left(A_{\beta}\right)=\mathcal{D}(A)$ is the infinitesimal generator of an analytic and exponentially stable semigroup on $\mathbb{X}$ of type lower than $-\beta$.

The proof of Theorem 2.2 mainly relies on the application of the Fattorini criterion, see [1]. Let us recall the hypotheses of $[1]$.

$\left(\mathcal{H}_{1}\right)$ The spectrum of $A$ consists of isolated eigenvalues with finite algebraic multiplicity.

$\left(\mathcal{H}_{2}\right)$ The family of root vectors of $A$ is complete in $\mathbb{X}$.

$\left(\mathcal{H}_{3}\right)$ The semigroup $\left(e^{t A}\right)$ is analytic.

Then [1, Theorem 1.3] states as follows:

Theorem 2.3. Assume $\left(\mathcal{H}_{1}\right)-\left(\mathcal{H}_{3}\right)$. Then $(A, B)$ is approximately controllable if and only if

$$
A^{*} \xi=\mu \xi \quad \text { and } \quad B^{*} \xi=0 \Longrightarrow \xi=0 .
$$

Adding the following hypothesis on the spectrum of $A$, we can obtain a stabilization result.

$\left(\mathcal{H}_{4}\right)$ There exists $\beta_{0}>0$ such that the spectrum of $A$ has no cluster point in

$$
\left\{z \in \mathbb{C} ; \operatorname{Re} z>-\beta_{0}\right\} \text {. }
$$

More precisely, 1, Theorem 1.6] implies the following result:

Theorem 2.4. Assume $\left(\mathcal{H}_{1}\right),\left(\mathcal{H}_{3}\right)$ and $\left(\mathcal{H}_{4}\right)$. Let us consider $\beta \in\left(0, \beta_{0}\right)$ and

$$
K:=\max \{\operatorname{dim} \operatorname{ker}(\mu \mathrm{I}-A) ; \mu \in \sigma(A), \operatorname{Re}(\mu) \geqslant-\beta\}<\infty .
$$

Assume

$$
\forall \mu \in \mathbb{C}, \operatorname{Re} \mu \geqslant-\beta, \quad A^{*} \xi=\mu \xi \quad \text { and } \quad B^{*} \xi=0 \Longrightarrow \xi=0 .
$$

Then, there exists $F_{\beta} \in \mathcal{L}(\mathbb{X}, \mathbb{U})$ with $\operatorname{rank}\left(F_{\beta}\right)=K$ such that $A_{\beta}:=A+B F_{\beta}$ with domain $\mathcal{D}\left(A_{\beta}\right)=\mathcal{D}(A)$ is the infinitesimal generator of an analytic and exponentially stable semigroup on $\mathbb{X}$ of type lower than $-\beta$.

Remark 2.5. In fact [1, Theorem 1.6] is stated in the case where there is no cluster point in the spectrum of $A$, but the proof of [1, Theorem 1.6] implies the above result. 
In order to prove Theorem 2.2 , let us start by studying the spectral properties of $A^{*}$. With the properties of $A_{0}$, the spectrum of $A_{0}$ is reduced to a nondecreasing sequence of eigenvalues $\left\{\Lambda_{k}\right\}_{k \geqslant 1} \subset \mathbb{R}_{+}^{*}$ such that $\Lambda_{k} \rightarrow \infty$ as $k \rightarrow \infty$. Moreover, there exists a corresponding orthonormal basis of $\mathbb{H}$ composed of eigenvectors $w_{k}$ with

$$
A_{0} w_{k}=\Lambda_{k} w_{k} \quad(k \geqslant 1) .
$$

Using the above notation, we can obtain the eigenvalues of $A^{*}$. More precisely, let us define

$$
\begin{gathered}
\delta_{k}:=\left(\lambda+\eta \Lambda_{k}\right)^{2}-4 \Lambda_{k}(\eta \lambda+\kappa), \\
K_{-}:=\left\{k \geqslant 1 ; \delta_{k}<0\right\}, \quad K_{0}:=\left\{k \geqslant 1 ; \delta_{k}=0\right\}, \quad K_{+}:=\left\{k \geqslant 1 ; \delta_{k}>0\right\} .
\end{gathered}
$$

Note that, since $\Lambda_{k} \rightarrow \infty, K_{-}$and $K_{0}$ are finite sets whereas $K_{+}$is an infinite set.

Then we write

$$
\begin{aligned}
& \mu_{0}:=0 \\
& \mu_{k}^{1}:=\frac{-\left(\lambda+\eta \Lambda_{k}\right)+i \sqrt{-\delta_{k}}}{2}, \quad \mu_{k}^{2}:=\frac{-\left(\lambda+\eta \Lambda_{k}\right)-i \sqrt{-\delta_{k}}}{2}, \quad\left(k \in K_{-}\right) \\
& \mu_{k}:=\frac{-\left(\lambda+\eta \Lambda_{k}\right)}{2}, \quad\left(k \in K_{0}\right),
\end{aligned}
$$

and

$$
\mu_{k}^{1}:=\frac{-\left(\lambda+\eta \Lambda_{k}\right)+\sqrt{\delta_{k}}}{2}, \quad \mu_{k}^{2}:=\frac{-\left(\lambda+\eta \Lambda_{k}\right)-\sqrt{\delta_{k}}}{2}, \quad\left(k \in K_{+}\right) .
$$

One can check that

$$
\begin{gathered}
\mu_{k}<0 \quad\left(k \in K_{0}\right), \quad \operatorname{Re} \mu_{k}^{j}<0 \quad\left(k \in K_{-} \cup K_{+}, j=1,2\right), \\
\mu_{k}+\lambda \neq 0 \quad\left(k \in K_{0}\right), \quad \mu_{k}^{j}+\lambda \neq 0 \quad\left(k \in K_{-} \cup K_{+}, j=1,2\right), \\
\lim _{k \rightarrow \infty} \mu_{k}^{1}=-\left(\lambda+\frac{\kappa}{\eta}\right), \quad \lim _{k \rightarrow \infty} \mu_{k}^{2}=-\infty .
\end{gathered}
$$

We also define

$$
\begin{gathered}
\xi_{0}^{* j}=\left[\begin{array}{c}
\lambda A_{0}^{-1} C_{0}^{*} e_{j} \\
-C_{0}^{*} e_{j} \\
(\lambda \eta+\kappa) e_{j}
\end{array}\right] \quad(1 \leqslant j \leqslant N), \quad \xi_{k}^{* j}=\left[\begin{array}{c}
\lambda+\mu_{k}^{j} \\
-\Lambda_{k} \\
0
\end{array}\right] w_{k} \quad\left(k \in K_{-} \cup K_{+}, j=1,2\right), \\
\xi_{k}^{* 1}=\left[\begin{array}{c}
\lambda+\mu_{k} \\
-\Lambda_{k} \\
0
\end{array}\right] w_{k}, \quad \xi_{k}^{* 2}=\left[\begin{array}{c}
0 \\
\left(\lambda+\mu_{k}\right) / \kappa \\
0
\end{array}\right] w_{k} \quad\left(k \in K_{0}\right) .
\end{gathered}
$$

Then we have the following property

Proposition 2.6. The eigenvalues of $A^{*}$ are given by

$$
\mu_{0}, \quad \mu_{k}^{j} \quad\left(k \in K_{-} \cup K_{+}, j=1,2\right), \quad \mu_{k} \quad\left(k \in K_{0}\right) .
$$

A corresponding family of generalized eigenvectors of $A^{*}$ associated with the above eigenvalues is given by 2.20, 2.21. More precisely, we have the following formula

$$
\begin{gathered}
A^{*} \xi_{0}^{* j}=0 \quad(1 \leqslant j \leqslant N), \quad A^{*} \xi_{k}^{* j}=\mu_{k}^{j} \xi_{k}^{* j} \quad\left(k \in K_{-} \cup K_{+}, j=1,2\right), \\
A^{*} \xi_{k}^{* 1}=\mu_{k} \xi_{k}^{* 1}, \quad A^{*} \xi_{k}^{* 2}=\mu_{k} \xi_{k}^{* 2}+\xi_{k}^{* 1}, \quad\left(k \in K_{0}\right) .
\end{gathered}
$$

If $A^{*} \xi^{*}=\mu \xi^{*}$, then $\xi^{*}$ is a linear combination of

$$
\xi_{0}^{* j}(1 \leqslant j \leqslant N), \quad \xi_{k}^{* j}\left(k \in K_{-} \cup K_{+}, j=1,2\right), \quad \text { and } \quad \xi_{k}^{* 1}\left(k \in K_{0}\right) .
$$

Proof. Assume that

$$
A^{*}\left[\begin{array}{l}
\phi \\
\psi \\
\alpha
\end{array}\right]=\mu\left[\begin{array}{l}
\phi \\
\psi \\
\alpha
\end{array}\right] .
$$

First assume that $\mu \neq 0$. Then we have $\alpha=0, \kappa \psi=\mu \phi+\eta A_{0} \phi$ and

$$
-(\eta(\lambda+\mu)+\kappa) A_{0} \phi=\mu(\lambda+\mu) \phi .
$$


- Case 1: $\phi=0$. Then $[\phi, \psi, \alpha]=0$.

- Case 2: $\phi \neq 0$ and $\eta(\lambda+\mu)+\kappa=0$. Then $\mu(\lambda+\mu)=0$ and since $\mu \neq 0, \kappa>0$, this leads to a contradiction.

- Case 3: $\phi \neq 0$ and $\eta(\lambda+\mu)+\kappa \neq 0$. Then, from 2.10, we deduce that $\mu$ satisfy for some $k \geqslant 1$ the equation

$$
(\mu+\lambda)\left(\mu+\eta \Lambda_{k}\right)+\kappa \Lambda_{k}=0 .
$$

Then solving this equation in $\mu$ leads to formula 2.11), 2.14 - 2.16). Standard computation yields that $[\phi, \psi, \alpha]$ is a linear combination of

$$
\xi_{k}^{* j}\left(k \in K_{-} \cup K_{+}, j=1,2\right), \quad \text { and } \quad \xi_{k}^{* 1}\left(k \in K_{0}\right) .
$$

Second, assume that $\mu=0$ in 2.25 . Then, if $\alpha=0$, we deduce that $[\phi, \psi, \alpha]=0$. Else, we can check that $[\phi, \psi, \alpha]$ is a linear combination of $\xi_{0}^{* j}(1 \leqslant j \leqslant N)$ given by 2.20 .

Similarly, we can define

$$
\begin{gathered}
\xi_{0}^{j}=\left[\begin{array}{c}
0 \\
0 \\
e_{j}
\end{array}\right] \quad(1 \leqslant j \leqslant N), \quad \xi_{k}^{j}=\left[\begin{array}{c}
\overline{\mu_{k}^{j}}\left(\lambda+\overline{\mu_{k}^{j}}\right) w_{k} \\
\kappa \overline{\mu_{k}^{j}} w_{k} \\
\left(\lambda+\overline{\mu_{k}^{j}}\right) C_{0} w_{k}
\end{array}\right] \quad\left(k \in K_{-} \cup K_{+}, j=1,2\right), \\
\xi_{k}^{1}=\left[\begin{array}{c}
\mu_{k}\left(\lambda+\mu_{k}\right) w_{k} \\
\kappa \mu_{k} w_{k} \\
\left(\lambda+\mu_{k}\right) C_{0} w_{k}
\end{array}\right], \quad \xi_{k}^{2}=\left[\begin{array}{c}
\left(\lambda+\mu_{k}\right) w_{k} \\
(\kappa \lambda)\left(\lambda+\mu_{k}\right)^{-1} w_{k} \\
0
\end{array}\right] \quad\left(k \in K_{0}\right) .
\end{gathered}
$$

Then standard computation shows that

$$
\begin{gathered}
A \xi_{0}^{j}=0 \quad j=1, \ldots, N, \quad A \xi_{k}^{j}=\overline{\mu_{k}^{j}} \xi_{k}^{j} \quad\left(k \in K_{-} \cup K_{+}, j=1,2\right), \\
A \xi_{k}^{1}=\mu_{k} \xi_{k}^{1}, \quad A \xi_{k}^{2}=\mu_{k} \xi_{k}^{2}+\xi_{k}^{1}, \quad\left(k \in K_{0}\right),
\end{gathered}
$$

Moreover we have the following result:

Proposition 2.7. The family of root vectors of $A$ is complete:

$$
\operatorname{span}\left(\left\{\xi_{k}^{j}\right\}_{k \geqslant 1, j=1,2} \cup\left\{\xi_{0}^{j}\right\}_{j=1, \ldots, N}\right)
$$

is dense in $\mathbb{X}$.

Proof. Assume $[f, g, \alpha] \in \mathbb{Y}$ is such that

$$
\left\langle[f, g, \alpha], \xi_{k}^{j}\right\rangle=0 \quad(k \geqslant 1, j=1,2), \quad\left\langle[f, g, \alpha], \xi_{0}^{j}\right\rangle=0 \quad(j=1, \ldots, N) .
$$

From (2.27), we deduce that $\alpha=0$.

Now, we decompose $f, g$ in the orthogonal basis $\left(w_{k}\right)$ :

$$
f=\sum_{k \geqslant 1} f_{k} w_{k}, \quad g=\sum_{k \geqslant 1} g_{k} w_{k},
$$

and we deduce from 2.27 and 2.28 the following relations:

$$
\begin{gathered}
{\left[\begin{array}{c}
\overline{\mu_{k}^{j}}\left(\lambda+\overline{\mu_{k}^{j}}\right) \\
\kappa \overline{\mu_{k}^{j}}
\end{array}\right] \cdot\left[\begin{array}{l}
f_{k} \\
g_{k}
\end{array}\right]=0 \quad\left(k \in K_{-} \cup K_{+}, j=1,2\right),} \\
{\left[\begin{array}{c}
\mu_{k}\left(\lambda+\mu_{k}\right) \\
\kappa \mu_{k}
\end{array}\right] \cdot\left[\begin{array}{c}
f_{k} \\
g_{k}
\end{array}\right]=0, \quad\left[\begin{array}{c}
\left(\lambda+\mu_{k}\right) \\
(\kappa \lambda)\left(\lambda+\mu_{k}\right)^{-1}
\end{array}\right] \cdot\left[\begin{array}{l}
f_{k} \\
g_{k}
\end{array}\right]=0 \quad\left(k \in K_{0}\right) .}
\end{gathered}
$$

Using (2.14)-2.16), we deduce $f_{k}=g_{k}=0$ for all $k \geqslant 1$.

We are now in a position to prove Theorem 2.2 
Proof of Theorem 2.2. We apply Theorem 2.3 (that is [1, Theorem 1.3]). First, since $\left(A_{0}, B_{0}\right)$ is approximately controllable and satisfies $\left(\mathcal{H}_{1}\right)-\left(\mathcal{H}_{3}\right)$, we deduce that $B_{0}^{*} w_{k} \neq 0$ for all $k \geqslant 1$.

Now, from Proposition 2.1. Proposition 2.6 and Proposition 2.7 $(A, B)$ satisfies $\left(\mathcal{H}_{1}\right)-\left(\mathcal{H}_{3}\right)$. We can thus apply again Theorem 2.3 and use the Fattorini criterion to prove the approximate controllability of $(2.2)$.

From 2.4, 2.20-2.21 and 2.18, we have

$$
\begin{gathered}
B^{*} \xi_{0}^{* j}=\lambda B_{0}^{*} A_{0}^{-1} C_{0}^{*} e_{j} \neq 0 \quad j=1, \ldots, N, \\
B^{*} \xi_{k}^{* j}=\left(\lambda+\mu_{k}^{j}\right) B_{0}^{*} w_{k} \neq 0 \quad\left(k \in K_{+} \cup K_{-}, j=1,2\right), \\
B^{*} \xi_{k}^{* 1}=\left(\lambda+\mu_{k}\right) B_{0}^{*} w_{k} \neq 0 \quad\left(k \in K_{0}\right) .
\end{gathered}
$$

Using Proposition 2.6 we deduce that 2.8 holds true and that $(A, B)$ is approximately controllable. 2.19

To obtain the stabilizability result, we apply Theorem [2.4 (that is, [1, Theorem 1.6]) combined with

\section{Case of multiple modes}

We can generalize the result obtained in Section 2 . Let us consider the same hypotheses on $\mathbb{H}, \mathbb{U}, A_{0}, B_{0}$ and $C_{0}$. We consider $\eta>0, M \in \mathbb{N}^{*}, \lambda_{i}, \kappa_{i} \in \mathbb{R}_{+}^{*}\left(i \in\{1, \ldots, M\}\right.$. We assume that $\lambda_{i}$ are distinct and for instance we assume

$$
0<\lambda_{1}<\ldots<\lambda_{M} .
$$

We consider the following generalization of 2.2 :

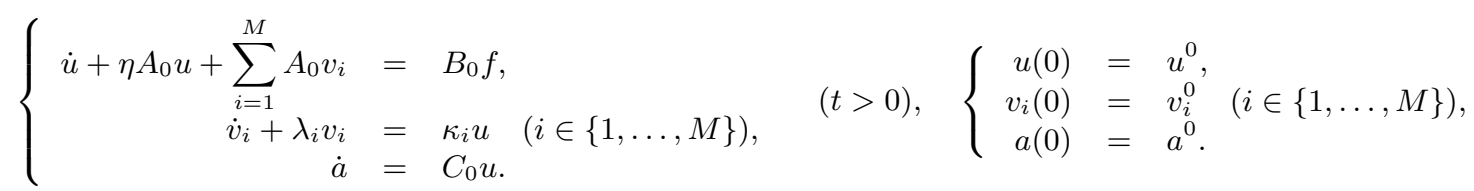

Setting $Z=\left[u, v_{1}, \ldots, v_{M}, a\right]$, we can write 3.2 under the form

$$
\dot{Z}=A Z+B f, \quad t>0, \quad Z(0)=Z^{0}
$$

by defining

$$
\begin{aligned}
& \mathbb{X}:=\mathbb{H} \times \mathbb{H}_{1}^{M} \times \mathbb{R}^{N}, \quad \mathcal{D}(A):=\mathbb{H}_{1} \times \mathbb{H}_{1}^{M} \times \mathbb{R}^{N}, \\
& A:=\left[\begin{array}{cccccc}
-\eta A_{0} & -A_{0} & -A_{0} & \cdots & -A_{0} & 0 \\
\kappa_{1} \mathrm{I} & -\lambda_{1} \mathrm{I} & 0 & \cdots & 0 & 0 \\
\kappa_{2} \mathrm{I} & 0 & -\lambda_{2} \mathrm{I} & \cdots & 0 & 0 \\
\vdots & \vdots & \vdots & \ddots & \vdots & \vdots \\
\kappa_{M} \mathrm{I} & 0 & 0 & \cdots & -\lambda_{M} \mathrm{I} & 0 \\
C_{0} & 0 & 0 & \cdots & 0 & 0
\end{array}\right], \quad B:=\left[\begin{array}{c}
B_{0} \\
0 \\
\vdots \\
0
\end{array}\right] \text {, }
\end{aligned}
$$

and

$$
Z=\left[u, v_{1}, \ldots, v_{M}, a\right], \quad Z^{0}=\left[u^{0}, v_{1}^{0}, \ldots, v_{M}^{0}, a^{0}\right]
$$

In order to state our main result, let us consider the function

$$
\mu \mapsto \eta+\sum_{j=1}^{M} \frac{\kappa_{j}}{\lambda_{j}+\mu} .
$$

One can check that it has exactly $M \operatorname{roots}\left(\mu^{j}\right)_{j=1}^{M}$ that are real and satisfy

$$
\mu^{M}<-\lambda_{M}<\mu^{M-1}<-\lambda_{M-1}<\cdots<\mu^{1}<-\lambda_{1} .
$$

The definitions of approximate controllability and stabilization are the same as in the previous section. Our main result in this section is the following theorem: 
Theorem 3.1. Assume $\left(A_{0}, B_{0}\right)$ is approximately controllable and that $B_{0}^{*} A_{0}^{-1} C_{0}^{*}: \mathbb{R}^{N} \rightarrow \mathbb{U}$ is injective. Then the system (3.2) is approximately controllable for any $T>0$.

Moreover, for any

$$
0<\beta<-\mu^{1}
$$

the system 3.2 is stabilizable in $\mathbb{X}$ with a rate lower than $-\beta$. More precisely, by denoting

$$
K:=\max \{\operatorname{dim} \operatorname{ker}(\mu \mathrm{I}-A) ; \mu \in \sigma(A), \operatorname{Re}(\mu) \geqslant-\beta\}<\infty,
$$

there exists $F_{\beta} \in \mathcal{L}(\mathbb{X}, \mathbb{U})$ with $\operatorname{rank}\left(F_{\beta}\right)=K$ such that $A_{\beta}:=A+B F_{\beta}$ with domain $\mathcal{D}\left(A_{\beta}\right)=\mathcal{D}(A)$ is the infinitesimal generator of an analytic and exponentially stable semigroup on $\mathbb{X}$ of type lower than $-\beta$.

The proof of Theorem 3.1 follows closely the proof of Theorem 2.2. First, following the proof of Proposition 2.1 we deduce

Proposition 3.2. The operator $(A, \mathcal{D}(A))$, defined by 3.3 -3.4 generates an analytic semigroup in $\mathbb{X}$. Its adjoint in $\mathbb{Y}:=\mathbb{H} \times \mathbb{H}_{-1}^{M} \times \mathbb{R}^{N}$ is given by

$$
\begin{aligned}
\mathcal{D}\left(A^{*}\right):= & \left.\left\{\phi, \psi_{1}, \ldots, \psi_{M}, \alpha\right] \in \mathbb{Y} ;-\eta A_{0} \phi+\sum_{j=1}^{M} \kappa_{j} \psi_{j} \in \mathbb{H}\right\}, \\
A^{*} & =\left[\begin{array}{cccccc}
-\eta A_{0} & \kappa_{1} \mathrm{I} & \kappa_{2} \mathrm{I} & \ldots & \kappa_{M} \mathrm{I} & C_{0}^{*} \\
-A_{0} & -\lambda_{1} \mathrm{I} & 0 & \cdots & 0 & 0 \\
-A_{0} & 0 & -\lambda_{2} \mathrm{I} & \cdots & 0 & 0 \\
\vdots & \vdots & \vdots & \ddots & \vdots & \vdots \\
-A_{0} & 0 & 0 & \cdots & -\lambda_{M} \mathrm{I} & 0 \\
0 & 0 & 0 & \cdots & 0 & 0
\end{array}\right] .
\end{aligned}
$$

Then, we use again the families $\left(\Lambda_{k}, w_{k}\right)$ associated with $A_{0}$ (see 2.10$)$ ), but we do not have here explicit formula such as 2.13 -2.16 . We have the following result:

Proposition 3.3. The eigenvalues of $A^{*}$ are $\mu_{0}=0$ and the roots $\mu_{k}^{j}(j=1, \ldots, M+1, k \geqslant 1)$ of the function

$$
\mu \mapsto \frac{\mu}{\Lambda_{k}}+\eta+\sum_{i=1}^{M} \frac{\kappa_{i}}{\mu+\lambda_{i}} .
$$

There exists $k_{0} \geqslant 1$ such that for $k \geqslant k_{0}$ these roots are real negative with

$$
\mu_{k}^{M+1}<-G<\mu_{k}^{M}<-\lambda_{M}<\mu_{k}^{M-1}<-\lambda_{M-1}<\cdots<\mu_{k}^{1}<-\lambda_{1},
$$

where $G>0$ is independent of $k$. We have

$$
\mu_{k}^{j} \rightarrow \mu^{j} \quad(j=1, \ldots, M), \quad \mu_{k}^{M+1} \sim-\eta \Lambda_{k} .
$$

The eigenvectors of $A^{*}$ are of the form

$$
\left[\phi,-\frac{A_{0} \phi}{\mu+\lambda_{1}}, \ldots,-\frac{A_{0} \phi}{\mu+\lambda_{M}}, 0\right]
$$

with $\phi$ an eigenvector of $A_{0}$ or

$$
\left[A_{0}^{-1} C_{0}^{*} \alpha,-\frac{C_{0}^{*} \alpha}{\lambda_{1}}, \ldots,-\frac{C_{0}^{*} \alpha}{\lambda_{M}},\left(\eta+\sum_{i=1}^{M} \frac{\kappa_{i}}{\lambda_{i}}\right) \alpha\right] .
$$

Finally, if $\mu_{k}^{j}=\mu_{k^{\prime}}^{j^{\prime}}$, then $\Lambda_{k}=\Lambda_{k^{\prime}}$ and in particular, the algebraic multiplicity of the eigenvalues of $A^{*}$ are finite. 
Proof. Assume that

$$
A^{*}\left[\begin{array}{c}
\phi \\
\psi_{1} \\
\vdots \\
\psi_{M} \\
\alpha
\end{array}\right]=\mu\left[\begin{array}{c}
\phi \\
\psi_{1} \\
\vdots \\
\psi_{M} \\
\alpha
\end{array}\right] .
$$

Case 1: assume that $\mu \neq 0$. Then we have $\alpha=0$. If there exists $i_{0} \in\{1, \ldots, M\}$ such that $\mu=-\lambda_{i_{0}}$, then $\left[\phi, \psi_{1}, \ldots, \psi_{M}, \alpha\right]=0$. We thus assume that for all $i \in\{1, \ldots, M\}, \mu+\lambda_{i} \neq 0$. Again, if $\phi=0$, then $\left[\phi, \psi_{1}, \ldots, \psi_{M}, \alpha\right]=0$. We thus obtain that

$$
\psi_{i}=-\frac{A_{0} \phi}{\mu+\lambda_{i}}
$$

and that there exists $k$ such that $\mu$ is a root of 3.9 and such that $\phi$ is an eigenvector associated with $\Lambda_{k}$.

In particular, $\mu$ is a root of the polynomial

$$
P_{k}(X):=\left(\frac{X}{\Lambda_{k}}+\eta\right) \prod_{i=1}^{M}\left(X+\lambda_{i}\right)+\sum_{i=1}^{M} \kappa_{i} \prod_{\ell \neq i}^{M}\left(X+\lambda_{\ell}\right) .
$$

One can check that $P_{k}$ admits a real root in the interval $\left(-\lambda_{i+1},-\lambda_{i}\right), i=1, \ldots, M-1$. Since $\Lambda_{k} \rightarrow \infty$, there exists $k_{0} \geqslant 1$ such that

$$
\Lambda_{k}>\max \left(\frac{2^{M+2}}{\eta^{2}} \sum_{i=1}^{M} \kappa_{i}, \frac{4 \lambda_{M}}{\eta}\right) \quad\left(k \geqslant k_{0}\right) .
$$

Let us consider $G$ such that

$$
\max \left(\frac{2^{M+1}}{\eta} \sum_{i=1}^{M} \kappa_{i}, 2 \lambda_{M}\right)<G<\frac{\eta \Lambda_{k_{0}}}{2} .
$$

With this choice, we can check that $P_{k}$ has a real root in the interval $\left(-G,-\lambda_{M}\right)$. In that case (that is for $\left.k \geqslant k_{0}\right)$, the last root of $P_{k}$ is also real and from the change of signs of $P_{k}$ in the intervals $\left(-\lambda_{i+1},-\lambda_{i}\right)$ and $\left(-G,-\lambda_{M}\right)$, we see that this last root belongs to $(-\infty,-G)$. We thus deduce 3.10 and the limits of $\mu_{k}^{j}$ for $j \leqslant M$.

For $\left(\mu_{k}^{M+1}\right)_{k \geqslant k_{0}}$, if it admits a bounded subsequence, we see that it converges towards a root of 3.5 so that for $k$ large enough, it belongs to one of the intervals $\left(-\lambda_{i+1},-\lambda_{i}\right)$ and $\left(-G,-\lambda_{M}\right)$ which is false. Therefore,

and from 3.9 , we deduce that

$$
\mu_{k}^{M+1} \rightarrow-\infty
$$

$$
\mu_{k}^{M+1} \sim-\eta \Lambda_{k}
$$

Case 2: assume $\mu=0$. Then standard computation yields (3.13).

One can check that the eigenvectors of $A$ are of the form

$$
[0,0, \ldots, 0, a], \quad a \in \mathbb{R}^{N}, a \neq 0
$$

for the eigenvalue $\mu=0$ and

$$
\left[w_{k}, \frac{\kappa_{1}}{\mu+\lambda_{1}} w_{k}, \ldots, \frac{\kappa_{M}}{\mu+\lambda_{M}} w_{k}, \frac{1}{\mu} C_{0} w_{k}\right]
$$

for the eigenvalues solutions of 3.9 . Using this, we can show the following result:

Proposition 3.4. The family of root vectors of $A$ is complete.

Proof. Assume $F=\left[f, g_{1}, g_{2}, \ldots, g_{M}, \alpha\right] \in \mathbb{Y}=\mathbb{H} \times \mathbb{H}_{-1}^{M} \times \mathbb{C}^{N}$ is such that

$$
\langle F, \xi\rangle=0
$$

for any $\xi$ root vector of $A$. 
We decompose $f$ and $g_{i}$ in the orthogonal basis $\left(w_{k}\right)$ :

$$
f=\sum_{k \geqslant 1} f_{k} w_{k}, \quad g_{i}=\sum_{k \geqslant 1} g_{i, k} w_{k}, \quad i=1,2, \ldots, M
$$

and we deduce from (3.17), that for $k \geqslant k_{0}$ (that is when we have 3.10 ),

$$
\left\langle\left[f_{k}, g_{1, k}, \ldots, g_{M, k}\right],\left[1, \frac{\kappa_{1}}{\mu_{k}^{j}+\lambda_{1}}, \ldots, \frac{\kappa_{M}}{\mu_{k}^{j}+\lambda_{M}}\right]\right\rangle=0, \quad \forall k \geqslant k_{0}, j=1,2, \ldots, M+1
$$

for the usual scalar product of $\mathbb{C}^{M+1}$. Noting that $\left[1, \frac{\kappa_{1}}{\mu_{k}^{j}+\lambda_{1}}, \ldots, \frac{\kappa_{M}}{\mu_{k}^{j}+\lambda_{M}}\right], j=1,2, \ldots, M+1$ are independent vectors of $\mathbb{C}^{M+1}$ for $k \geqslant k_{0}$, we deduce that

$$
F \in\left(\mathbb{W}_{k_{0}}\right)^{M+1} \times \mathbb{C}^{N}, \quad \text { where } \mathbb{W}_{k_{0}}=\operatorname{span}\left\{w_{k}, k<k_{0}\right\} .
$$

From the expression (3.4) of $A$, we see that it is a linear operator of the finite-dimensional subspace $\left(\mathbb{W}_{k_{0}}\right)^{M+1} \times \mathbb{C}^{N}$. From classical result of linear algebra, we deduce that the corresponding linear operator admits a basis of root vectors in $\left(\mathbb{W}_{k_{0}}\right)^{M+1} \times \mathbb{C}^{N}$ and thus we deduce that $F=0$.

Combining Proposition 3.2 . Proposition 3.3 and Proposition 3.4 and following the proof of Theorem 2.2 we can prove Theorem 3.1 in a completely similar way. We thus skip the corresponding proof.

\section{Proof of Theorem 1.1}

In order to show Theorem 1.1 we are going to apply Theorem 2.2 First we show that $(1.12--1.19$ can be written under the form $(2.2)$. We recall that $\mathbb{H}$ is defined by 1.20 . Now we define $A_{0}$ as follows:

$$
\begin{gathered}
\mathcal{D}\left(A_{0}\right)=\left\{u \in H_{0}^{1}(\Omega)|u|_{\mathcal{F}} \in H^{2}(\mathcal{F}), \operatorname{div} u=0 \text { in } \Omega, \mathbb{D}(u)=0 \text { in } \mathcal{S}\right\}, \\
\mathcal{A}_{0} u=\left\{\begin{array}{l}
-\Delta u \\
{\left[\frac{1}{m} \int_{\partial \mathcal{S}} 2 \mathbb{D}(u) n d \Gamma\right]+\left[J_{0}^{-1} \int_{\partial \mathcal{S}} \widetilde{y} \times 2 \mathbb{D}(u) n d \Gamma_{\widetilde{y}}\right] \times y \quad \text { in } \mathcal{S} \quad\left(u \in \mathcal{D}\left(A_{0}\right)\right),} \\
A_{0} u=\mathbb{P} \mathcal{A}_{0} u \quad\left(u \in \mathcal{D}\left(A_{0}\right)\right)
\end{array}\right.
\end{gathered}
$$

where $\mathbb{P}$ is the orthogonal projector from $L^{2}(\Omega)$ onto $\mathbb{H}$.

The operator $C_{0} \in \mathcal{L}\left(\mathbb{H}, \mathbb{R}^{6}\right)$ is defined by

$$
C_{0} u=\left(\ell_{u}, \omega_{u}\right) \quad \text { if } u(y)=\ell_{u}+\omega_{u} \times y \quad(y \in \mathcal{S}) .
$$

We also set $\mathbb{U}:=L^{2}(\mathcal{O})$ and the control operator $B_{0} \in \mathcal{L}\left(L^{2}(\mathcal{O}), \mathbb{H}\right)$ is given by

$$
B_{0} f=\mathbb{P}\left(f \chi_{\mathcal{O}}\right) \quad f \in \mathbb{U} .
$$

We set the initial conditions as:

$$
u^{0}=\left\{\begin{array}{ll}
u^{0} & \text { in } \mathcal{F} \\
\ell^{0}+\omega^{0} \times y & \text { in } \mathcal{S},
\end{array} \quad v^{0}=\left\{\begin{array}{ll}
v^{0} & \text { in } \mathcal{F} \\
k^{0}+r^{0} \times y & \text { in } \mathcal{S},
\end{array} \quad a 0=\left[h^{0}, \theta^{0}\right],\right.\right.
$$

where $u^{0}, v^{0}, \ell^{0}, \omega^{0}, k^{0}, r^{0}, h^{0}$ and $\theta^{0}$ are as given in $1.19 p$.

With this notation, we see that we can write $1.12-(1.19)$ as 2.2 , where $a=[h, \theta] \in \mathbb{R}^{6}$. We are now in a position to prove Theorem 1.1 
Proof of Theorem 1.1. We recall (see, for instance, 34, Proposition 5.3]) that $A_{0}$ is positive and self-adjoint. The couple $\left(A_{0}, B_{0}\right)$ is approximately controllable. Indeed, by using the Fattorini criterion (that is Theorem 2.3), we only need to show that if

$$
\left\{\begin{array}{c}
-\Delta u+\nabla p=\mu u \quad \text { in } \mathcal{F}, \\
\operatorname{div} u=0 \quad \text { in } \mathcal{F}, \\
u=0 \quad \text { on } \partial \Omega, \\
u(y)=\ell+\omega \times y \quad y \in \partial \mathcal{S}, \\
m \mu \ell=\int_{\partial \mathcal{S}} \Sigma_{1}(u, p) n d \Gamma, \\
J_{0} \mu \omega=\int_{\partial \mathcal{S}} y \times \Sigma_{1}(u, p) n d \Gamma,
\end{array}\right.
$$

and if $B_{0}^{*} u=u_{\mid \mathcal{O}} \equiv 0$, then $u \equiv 0$. In the above system,

$$
\Sigma_{1}(u, p)=2 \mathbb{D}(u)-p \mathbb{I}_{3} .
$$

By the standard unique continuation property on the Stokes system (see [11), then $u \equiv 0$ in $\mathcal{F}$ and thus $\ell=\omega=0$ by using the trace of $u$ on $\partial \mathcal{S}$.

It remains to show that $B_{0}^{*} A_{0}^{-1} C_{0}^{*}: \mathbb{R}^{6} \rightarrow \mathbb{U}$ is injective. We endow $\mathbb{R}^{6}$ with the scalar product

$$
\left\langle(k, r),\left(k^{\prime}, r^{\prime}\right)\right\rangle=m k \cdot k^{\prime}+J_{0} r \cdot r^{\prime}
$$

Then, using 1.10 and 1.11

$$
\left\langle(k, r),\left(k^{\prime}, r^{\prime}\right)\right\rangle=\int_{\mathcal{S}} \rho_{\mathcal{S}}(k+r \times y) \cdot\left(k^{\prime}+r^{\prime} \times y\right) d y .
$$

It yields that if $\xi=(k, r) \in \mathbb{R}^{6}$, then

$$
C_{0}^{*} \xi=\mathbb{P}\left[(k+r \times y) \chi_{\mathcal{S}}\right],
$$

where $\chi_{\mathcal{S}}$ is the characteristic function of $\mathcal{S}$.

Thus, with 4.1 4.3 , we deduce that if $B_{0}^{*} A_{0}^{-1} C_{0}^{*} \xi=0$, then

$$
\left\{\begin{array}{c}
-\Delta u+\nabla p=0 \quad \text { in } \mathcal{F}, \\
\operatorname{div} u=0 \quad \text { in } \mathcal{F}, \\
u=0 \quad \text { on } \partial \Omega, \\
u(y)=\ell+\omega \times y \quad y \in \partial \mathcal{S}, \\
m k=\int_{\partial \mathcal{S}} \Sigma_{1}(u, p) n d \Gamma, \\
J_{0} r=\int_{\partial \mathcal{S}} y \times \Sigma_{1}(u, p) n d \Gamma, \\
u \equiv 0 \quad \text { in } \mathcal{O} .
\end{array}\right.
$$

By using again the unique continuation property of the Stokes system, then $u \equiv 0$ in $\mathcal{F}$ and $\nabla p \equiv 0$ in $\mathcal{F}$. Thus $k=r=0$ and we deduce the injectivity of $B_{0}^{*} A_{0}^{-1} C_{0}^{*}$. Applying Theorem 2.2, we deduce Theorem 1.1

\section{Lack of null controllability}

The goal of this section is to show that if $\mathcal{O}$ is a proper open subset of $\mathcal{F}$ then there exists an initial condition $\left[u^{0}, v^{0},\left(h^{0}, \theta^{0}\right)\right] \in \mathbb{H}_{1 / 2} \times \mathbb{H}_{1} \times \mathbb{R}^{6}$ such that for any finite time $T>0$ and for any control $f$, the solution $[u, v,(\ell, \omega),(k, r),(h, \theta)]$ of $(1.12)-(1.19)$ cannot be driven to 0 for the fluid velocity.

The proof is based on a localization procedure in the fluid and thus can be deduced from results obtained in 21]. The statement is the following one: 
Theorem 5.1. Assume $\mathcal{O}$ is an open subset of $\mathcal{F}, \mathcal{O} \neq \mathcal{F}$. There exists $\left[u^{0}, v^{0},\left(h^{0}, \theta^{0}\right)\right] \in \mathbb{H}_{1 / 2} \times \mathbb{H}_{1} \times \mathbb{R}^{6}$ such that for any $T>0$ and for any $f \in L^{2}\left(0, T ; L^{2}(\mathcal{O})\right)$, the solution $[u, v,(\ell, \omega),(k, r),(h, \theta)]$ of 1.12 - 1.19 satisfies

$$
u(T, \cdot) \not \equiv 0 .
$$

In order to prove the above result, we have the following results:

Proposition 5.2. Assume $\mathcal{O}$ is an open subset of $\mathcal{F}, \mathcal{O} \neq \mathcal{F}$. and let us consider $f \in L^{2}\left(0, T ; L^{2}(\mathcal{O})\right)$. Then the solution of $[u, v,(\ell, \omega),(k, r),(h, \theta)]$ of $1.12-1.19$ with $u^{0} \equiv 0, v^{0} \equiv 0$ satisfies

$$
u, v \in C^{\infty}((0, T] \times(\mathcal{F} \backslash \overline{\mathcal{O}})) .
$$

Proof. The proof is based on Theorem 3.3 and the proof of Theorem 3.1 in 21. First, from Proposition 2.1 we deduce that

$$
u \in H^{1}\left(0, T ; L^{2}(\mathcal{F})\right) \cap L^{2}\left(0, T ; H^{2}(\mathcal{F})\right) \cap C^{0}\left([0, T] ; H^{1}(\mathcal{F})\right), \quad v \in H^{1}\left(0, T ; H^{2}(\mathcal{F})\right) .
$$

Second, let us consider $x_{0} \in \mathcal{F} \backslash \overline{\mathcal{O}}$ and $r_{0}>r_{1}>0$ with $B\left(x_{0}, r_{0}\right) \subset \mathcal{F} \backslash \overline{\mathcal{O}}$. We also take $\tilde{\chi}_{0}$ a smooth function with support in $B\left(x_{0}, r_{0}\right)$ and equal to 1 in $B\left(x_{0}, r_{1}\right)$. Then we deduce from $1.12-1.19$ that

$$
\vartheta=\tilde{\chi}_{0} \operatorname{curl} u, \quad \zeta=\tilde{\chi}_{0} \operatorname{curl} v,
$$

satisfy

where

$$
\left\{\begin{array}{c}
\frac{\partial \vartheta}{\partial t}-\eta \Delta \vartheta-\Delta \zeta=F \quad \text { in }(0, T) \times B\left(x_{0}, r_{0}\right), \\
\frac{\partial \zeta}{\partial t}+\lambda \zeta=\kappa \vartheta \quad \text { in }(0, T) \times B\left(x_{0}, r_{0}\right), \\
\vartheta=\zeta=0 \quad \text { on }(0, T) \times \partial B\left(x_{0}, r_{0}\right), \\
\vartheta(0, \cdot)=\zeta(0, \cdot)=0 \quad \text { in } B\left(x_{0}, r_{0}\right),
\end{array}\right.
$$

\[ F=-2(\eta(\nabla \operatorname{curl} u)+(\nabla \operatorname{curl} v)) \nabla \chi_{0}-\Delta \chi_{0}(\eta \operatorname{curl} u+\operatorname{curl} \widetilde{v}) \]
Using again Proposition 2.1 or directly Theorem 3.3 in 21, we deduce that

$$
\vartheta \in H^{1}\left(0, T ; L^{2}\left(B\left(x_{0}, r_{0}\right)\right)\right) \cap L^{2}\left(0, T ; H^{2}\left(B\left(x_{0}, r_{0}\right)\right)\right), \quad \zeta \in H^{1}\left(0, T ; H^{2}\left(B\left(x_{0}, r_{0}\right)\right)\right) .
$$

Proceeding by induction as in the proof of of Theorem 3.1 in 21] (here we see that the equations of the rigid body are not used), we can show that that

$$
\vartheta, \zeta \in C^{\infty}\left((0, T] \times B\left(x_{0}, r\right)\right)
$$

for some $r \in\left(0, r_{1}\right)$.

Then using that

$$
-\Delta u=\operatorname{curl} \vartheta \quad \text { and } \quad-\Delta v=\operatorname{curl} \zeta \text { in } B\left(x_{0}, r\right),
$$

we deduce the result from the interior regularity of the laplacian (see, for instance, [10, Theorem 3, p.334]).

Then, we have the following result:

Proposition 5.3. Assume $\mathcal{O}$ is an open subset of $\mathcal{F}, \mathcal{O} \neq \mathcal{F}$. and let us consider $x_{0} \in \mathcal{F} \backslash \overline{\mathcal{O}}$. Then there exists $\left[u^{0}, v^{0},\left(h^{0}, \theta^{0}\right)\right] \in \mathbb{H}_{1 / 2} \times \mathbb{H}_{1} \times \mathbb{R}^{6}$ such that the solution $[u, v,(\ell, \omega),(k, r),(h, \theta)]$ of 1.12 - 1.19 with $f \equiv 0$ satisfies that for any $t>0, u(t, \cdot)$ is not $C^{\infty}$ at $x_{0}$.

Proof. We start with the system

$$
\left\{\begin{array}{c}
\frac{\partial \vartheta}{\partial t}-\eta \Delta \vartheta-\Delta \zeta=0 \quad \text { in } \mathbb{R}_{+}^{*} \times \mathbb{R}^{3}, \\
\frac{\partial \zeta}{\partial t}+\lambda \zeta=\kappa \vartheta \quad \text { in } \mathbb{R}_{+}^{*} \times \mathbb{R}^{3}, \\
\vartheta(0, \cdot)=\vartheta^{0}, \quad \zeta(0, \cdot)=\zeta^{0} \text { in } \mathbb{R}^{3} .
\end{array}\right.
$$


From Theorem 3.5 in 21], there exists $u_{*}^{0} \in H^{1}\left(\mathbb{R}^{3}\right)$ and $v_{*}^{0} \in H^{2}\left(\mathbb{R}^{3}\right)$ such that the solution of the above system with $\vartheta^{0}=\operatorname{curl} u_{*}^{0}$ and $\zeta^{0}=\operatorname{curl} v_{*}^{0}$ satisfies for any $t>0$,

$$
\vartheta(t, \cdot), \zeta(t, \cdot) \in C^{\infty}\left(\mathbb{R}^{3} \backslash\left\{x_{0}\right\}\right)
$$

and $\vartheta(t, \cdot)$ is not $C^{\infty}$ at $x_{0}$.

Then, we apply Lemma 3.7 in 21 to deduce the existence of $u^{0} \in H_{0}^{1}(\mathcal{F})$ and $v^{0} \in H^{2}(\mathcal{F}) \cap H_{0}^{1}(\mathcal{F})$, with $\operatorname{div} u^{0}=\operatorname{div} v^{0}=0$ such that

$$
\operatorname{curl} u^{0}=\vartheta^{0} \text { and } \operatorname{curl} v^{0}=\zeta^{0}
$$

in a neighborhood of $x_{0}$. Extending $u^{0}$ and $v^{0}$ by 0 in $\mathcal{S}$, we deduce that $u^{0} \in \mathbb{H}_{1 / 2}$ and $v^{0} \in \mathbb{H}_{1}$. Taking $\left(h^{0}, \theta^{0}\right) \in \mathbb{R}^{6}$ arbitrarily we can thus consider the solution $[u, v,(\ell, \omega),(k, r),(h, \theta)]$ of 1.12 - $(1.19$ with $f \equiv 0$. Then

$$
\widetilde{\vartheta}:=\operatorname{curl} u-\vartheta, \quad \widetilde{\zeta}:=\operatorname{curl} v-\zeta
$$

satisfy

$$
\left\{\begin{array}{c}
\frac{\partial \widetilde{\vartheta}}{\partial t}-\eta \Delta \widetilde{\vartheta}-\Delta \widetilde{\zeta}=0 \quad \text { in }(0, T) \times B\left(x_{0}, r\right), \\
\frac{\partial \widetilde{\zeta}}{\partial t}+\lambda \widetilde{\zeta}=\kappa \widetilde{\vartheta} \quad \text { in }(0, T) \times B\left(x_{0}, r\right), \\
\widetilde{\vartheta}=g_{1}, \quad \widetilde{\zeta}=g_{2} \quad \text { on }(0, T) \times \partial B\left(x_{0}, r\right), \\
\widetilde{\vartheta}(0, \cdot)=\widetilde{\zeta}(0, \cdot)=0 \quad \text { in } B\left(x_{0}, r\right),
\end{array}\right.
$$

for $r>0$ small enough, where $g_{1}=\left.(\operatorname{curl} u-\vartheta)\right|_{(0, T) \times \partial B\left(x_{0}, r\right)}$ and $g_{2}=\left.(\operatorname{curl} v-\zeta)\right|_{(0, T) \times \partial B\left(x_{0}, r\right)}$. As for 5.2 , one can show that

$$
\widetilde{\vartheta}, \widetilde{\zeta} \in C^{\infty}\left((0, T] \times B\left(x_{0}, \widetilde{r}\right)\right)
$$

for $\widetilde{r}$ small enough. This yields the result.

From the above two propositions, we can deduce Theorem 5.1

Proof of Theorem 5.1. We consider the initial condition $\left[u^{0}, v^{0},\left(h^{0}, \theta^{0}\right)\right] \in \mathbb{H}_{1 / 2} \times \mathbb{H}_{1} \times \mathbb{R}^{6}$ of Proposition 5.3 and we assume $f \in L^{2}\left(0, T ; L^{2}(\mathcal{O})\right.$. We can then decompose the corresponding solution $[u, v,(\ell, \omega),(k, r),(h, \theta)]$ of 1.12)-(1.19) into a solution with null initial conditions and a solution with null source. Gathering Proposition 5.2 and Proposition 5.3 we deduce that $u(T, \cdot)$ is not $C^{\infty}$ at $x_{0}$ and thus is not null.

\section{Some extensions of Theorem 1.1}

Using the proof and the framework of Section 4 to obtain Theorem 1.1 , we see that we can deduce several extensions of Theorem 1.1

For instance, we can obtain the approximate controllability and stabilization of $1.12-1.19$ with a control $f$ with one component that cancels. For instance, we assume that $f_{3}=0$ and we thus control the fluid-structure system with only two scalar controls $\left(f_{1}\right.$ and $\left.f_{2}\right)$. We thus replace the equation 1.12 by

$$
\left\{\begin{array}{c}
\frac{\partial u}{\partial t}-\operatorname{div}(\Sigma(u, p)+2 \mathbb{D}(v))=\left(f_{1}, f_{2}, 0\right) \chi_{\mathcal{O}} \\
\operatorname{div} u=0 \quad t>0, y \in \mathcal{F},
\end{array} \quad t>0, y \in \mathcal{F},\right.
$$

Theorem 6.1. Assume that $\mathcal{O}$ is a nonempty open subset of $\mathcal{F}$. Then the system (6.1), (1.13)-(1.19) is approximately controllable. Assume moreover that

$$
0<\beta<\lambda+\frac{\kappa}{\eta}
$$

Then the system (6.1), 1.13)-1.19) is exponentially stabilizable with rate lower than $-\beta$ and with a feedback of finite dimension. 
Proof. The proof is completely similar to the proof of Theorem 1.1 we only replace 4.5 by

$$
B_{0}\left(f_{1}, f_{2}\right)=\mathbb{P}\left(\left(f_{1}, f_{2}, 0\right) \chi_{\mathcal{O}}\right), \quad\left(f_{1}, f_{2}\right) \in \mathbb{U} .
$$

Then, applying the Fattorini criterion to show that $\left(A_{0}, B_{0}\right)$ is approximately controllable, we consider a solution $(u, p)$ of 4.7 with $B_{0}^{*} u=\left(u_{1 \mid \mathcal{O}}, u_{2 \mid \mathcal{O}}\right) \equiv 0$. Combining this with $\operatorname{div} u=0$, we deduce

$$
\frac{\partial u}{\partial x_{3}} \equiv 0 \quad \text { in } \mathcal{O}
$$

Moreover, $\left(\frac{\partial u}{\partial x_{3}}, \frac{\partial p}{\partial x_{3}}\right)$ satisfies the following system:

$$
\left\{\begin{array}{cc}
-\Delta\left(\frac{\partial u}{\partial x_{3}}\right)+\nabla\left(\frac{\partial p}{\partial x_{3}}\right)=\lambda\left(\frac{\partial u}{\partial x_{3}}\right) & \text { in } \mathcal{F} \\
\operatorname{div}\left(\frac{\partial u}{\partial x_{3}}\right)=0 \quad \text { in } \mathcal{F}, &
\end{array}\right.
$$

and from 11, we deduce

$$
\frac{\partial u}{\partial x_{3}} \equiv 0 \quad \text { in } \mathcal{F} \text {. }
$$

By applying the Poincaré inequality, the above relation yields

$$
u \equiv 0 \quad \text { in } \mathcal{F}
$$

and thus $\ell=\omega=0$ by using the trace of $u$ on $\partial \mathcal{S}$.

The proof of the injectivity of $B_{0}^{*} A_{0}^{-1} C_{0}^{*}: \mathbb{R}^{6} \rightarrow \mathbb{U}$ is done similarly. Applying Theorem 2.2 we thus deduce Theorem 6.1

We can also obtain the approximate controllability and stabilization of a linear fluid-rigid body system for a fluid modeled by a linear Jeffreys model with several relaxation mode:

$$
\begin{aligned}
& \left\{\begin{aligned}
\frac{\partial u}{\partial t}-\operatorname{div}(\Sigma(u, p)+2 \mathbb{D}(v)) & =f \chi_{\mathcal{O}} \quad t>0, y \in \mathcal{F}, \\
\operatorname{div} u & =0 \quad t>0, y \in \mathcal{F},
\end{aligned}\right. \\
& \left\{\begin{array}{c}
u=0 \quad t>0, y \in \partial \Omega \\
u(t, y)=\ell(t)+\omega(t) \times y \quad t>0, y \in \partial \mathcal{S}
\end{array}\right. \\
& \left\{\begin{array}{c}
m \ell^{\prime}=-\int_{\partial \mathcal{S}}(\Sigma(u, p)+2 \mathbb{D}(v)) n d \Gamma \quad t>0, \\
J_{0} \omega^{\prime}=-\int_{\partial \mathcal{S}} y \times(\Sigma(u, p)+2 \mathbb{D}(v)) n d \Gamma \quad t>0,
\end{array}\right. \\
& \left\{\begin{array}{c}
\frac{\partial v_{i}}{\partial t}+\lambda_{i} v_{i}=\kappa_{i} u \quad t>0, y \in \mathcal{F}, i \in\{1, \ldots, M\} \\
\operatorname{div} v_{i}=0 \quad t>0, y \in \mathcal{F}, i \in\{1, \ldots, M\}
\end{array}\right. \\
& \left\{\begin{array}{c}
v_{i}=0 \quad t>0, y \in \partial \Omega, i \in\{1, \ldots, M\} \\
v_{i}(t, y)=k_{i}(t)+r_{i}(t) \times y \quad t>0, y \in \partial \mathcal{S}, i \in\{1, \ldots, M\}
\end{array}\right. \\
& \left\{\begin{array}{cc}
k_{i}^{\prime}+\lambda_{i} k_{i}=\kappa_{i} \ell & \forall t>0, i \in\{1, \ldots, M\} \\
r_{i}^{\prime}+\lambda_{i} r_{i}=\kappa_{i} \omega & \forall t>0, i \in\{1, \ldots, M\}
\end{array}\right. \\
& \begin{cases}h^{\prime}=\ell & \forall t>0 \\
\theta^{\prime}=\omega & \forall t>0\end{cases} \\
& \left\{\begin{array}{c}
u(0, \cdot)=u^{0}, \quad v_{i}(0, \cdot)=v_{i}^{0}, i \in\{1, \ldots, M\} \quad \text { in } \mathcal{F}, \\
h(0)=h^{0}, \quad \theta(0)=\theta^{0}, \quad \ell(0)=\ell^{0}, \quad \omega(0)=\omega^{0}, \quad k_{i}(0)=k_{i}^{0}, \quad r_{i}(0)=r_{i}^{0}, i \in\{1, \ldots, M\} .
\end{array}\right.
\end{aligned}
$$

Our main result is the following one

Theorem 6.2. Assume $\mathcal{O}$ is a nonempty open subset of $\mathcal{F}$. Assume also that $0<\lambda_{1}<\ldots<\lambda_{M}$. Then the system 6.3)-6.10 is approximately controllable. Assume moreover that

$$
0<\beta<-\mu^{1}
$$

where $\mu^{1}$ is the largest root of 3.5 . Then the system 6.3 -6.10 is exponentially stabilizable with rate lower than $-\beta$ and with a feedback of finite dimension. 


\section{Acknowledgments.}

Debanjana Mitra acknowledges the support from an Inspire Faculty Fellowship, RD/0118-DSTIN40-001. Arnab Roy was supported by the Czech Science Foundation (GAČR) project GA19-04243S. The Institute of Mathematics, CAS is supported by RVO:67985840. Takéo Takahashi was partially supported by the ANR research project IFSMACS (ANR-15-CE40-0010). The three authors were partially supported by the IFCAM project "Analysis, Control and Homogenization of Complex Systems".

\section{References}

[1] Mehdi Badra and Takéo Takahashi. On the Fattorini criterion for approximate controllability and stabilizability of parabolic systems. ESAIM Control Optim. Calc. Var., 20(3):924-956, 2014.

[2] José Luiz Boldrini, Anna Doubova, Enrique Fernández-Cara, and Manuel González-Burgos. Some controllability results for linear viscoelastic fluids. SIAM J. Control Optim., 50(2):900-924, 2012.

[3] Muriel Boulakia and Sergio Guerrero. Local null controllability of a fluid-solid interaction problem in dimension 3. J. Eur. Math. Soc. (JEMS), 15(3):825-856, 2013.

[4] Muriel Boulakia and Axel Osses. Local null controllability of a two-dimensional fluid-structure interaction problem. ESAIM Control Optim. Calc. Var., 14(1):1-42, 2008.

[5] Shirshendu Chowdhury, Debanjana Mitra, Mythily Ramaswamy, and Michael Renardy. Approximate controllability results for linear viscoelastic flows. J. Math. Fluid Mech., 19(3):529-549, 2017.

[6] Nicolae Cîndea, Sorin Micu, Ionel Rovenţa, and Marius Tucsnak. Particle supported control of a fluidparticle system. J. Math. Pures Appl. (9), 104(2):311-353, 2015.

[7] Anna Doubova and Enrique Fernández-Cara. Some control results for simplified one-dimensional models of fluid-solid interaction. Math. Models Methods Appl. Sci., 15(5):783-824, 2005.

[8] Anna Doubova and Enrique Fernández-Cara. On the control of viscoelastic Jeffreys fluids. Systems Control Lett., 61(4):573-579, 2012.

[9] Anna Doubova, Enrique Fernández-Cara, and Manuel González-Burgos. Controllability results for linear viscoelastic fluids of the Maxwell and Jeffreys kinds. C. R. Acad. Sci. Paris Sér. I Math., 331(7):537-542, 2000.

[10] Lawrence C. Evans. Partial differential equations, volume 19 of Graduate Studies in Mathematics. American Mathematical Society, Providence, RI, second edition, 2010.

[11] Caroline Fabre and Gilles Lebeau. Prolongement unique des solutions de l'equation de Stokes. Comm. Partial Differential Equations, 21(3-4):573-596, 1996.

[12] E. Fernández-Cara, Francisco Guillén, and Rubens R. Ortega. Mathematical modeling and analysis of viscoelastic fluids of the Oldroyd kind. In Handbook of numerical analysis, Vol. VIII, Handb. Numer. Anal., VIII, pages 543-661. North-Holland, Amsterdam, 2002.

[13] Karoline Götze. Strong solutions for the interaction of a rigid body and a viscoelastic fluid. J. Math. Fluid Mech., 15(4):663-688, 2013.

[14] Colette Guillopé and Jean-Claude Saut. Existence results for the flow of viscoelastic fluids with a differential constitutive law. Nonlinear Anal., 15(9):849-869, 1990.

[15] Oleg Imanuvilov and Takéo Takahashi. Exact controllability of a fluid-rigid body system. J. Math. Pures Appl. (9), 87(4):408-437, 2007.

[16] Oleg Imanuvilov and Takéo Takahashi. Exact controllability of a fluid-rigid body system. J. Math. Pures Appl. (9), 87(4):408-437, 2007.

[17] Günter Leugering. Exact controllability in viscoelasticity of fading memory type. Applicable Anal., 18(3):221-243, 1984.

[18] Günter Leugering. Exact boundary controllability of an integro-differential equation. Appl. Math. Optim., 15(3):223-250, 1987.

[19] Günter Leugering. Time optimal boundary controllability of a simple linear viscoelastic liquid. Math. Methods Appl. Sci., 9(3):413-430, 1987. 
[20] Yuning Liu, Takéo Takahashi, and Marius Tucsnak. Single input controllability of a simplified fluidstructure interaction model. ESAIM Control Optim. Calc. Var., 19(1):20-42, 2013.

[21] Debayan Maity, Debanjana Mitra, and Michael Renardy. Lack of null controllability of viscoelastic flows. ESAIM Control Optim. Calc. Var., 25:Paper No. 60, 26, 2019.

[22] Amnon Pazy. Semigroups of linear operators and applications to partial differential equations, volume 44 of Applied Mathematical Sciences. Springer-Verlag, New York, 1983.

[23] Mythily Ramaswamy, Arnab Roy, and Takéo Takahashi. Remark on the global null controllability for a viscous Burgers-particle system with particle supported control. Appl. Math. Lett., 107:106483, 7 pp., 2020.

[24] Jean-Pierre Raymond. Feedback stabilization of a fluid-structure model. SIAM J. Control Optim., 48(8):5398-5443, 2010.

[25] Jean-Pierre Raymond and Muthusamy Vanninathan. Exact controllability in fluid-solid structure: the Helmholtz model. ESAIM Control Optim. Calc. Var., 11(2):180-203, 2005.

[26] Michael Renardy. Mathematical analysis of viscoelastic flows, volume 73 of CBMS-NSF Regional Conference Series in Applied Mathematics. Society for Industrial and Applied Mathematics (SIAM), Philadelphia, PA, 2000.

[27] Michael Renardy. Are viscoelastic flows under control or out of control? Systems Control Lett., 54(12):1183-1193, 2005.

[28] Michael Renardy. On control of shear flow of an upper convected Maxwell fluid. ZAMM Z. Angew. Math. Mech., 87(3):213-218, 2007.

[29] Michael Renardy. Controllability of viscoelastic stresses for nonlinear Maxwell models. Journal of Non-Newtonian Fluid Mechanics, 156(1):70-74, 2009.

[30] Arnab Roy and Takéo Takahashi. Local null controllability of a rigid body moving into a Boussinesq flow. Math. Control Relat. Fields, 9(4):793-836, 2019.

[31] Arnab Roy and Takéo Takahashi. Stabilization of a rigid body moving in a compressible viscous fluid, 2019.

[32] Lamis Marlyn Kenedy Sabbagh. Study of rigid solids movement in a viscous fluid. Theses, Université Montpellier ; Université libanaise, November 2018. https://tel.archives-ouvertes.fr/ tel-02159446.

[33] Evgeny Savelev and Michael Renardy. Control of homogeneous shear flow of multimode Maxwell fluids. Journal of Non-Newtonian Fluid Mechanics, 165(3):136-142, 2010.

[34] Takéo Takahashi. Analysis of strong solutions for the equations modeling the motion of a rigid-fluid system in a bounded domain. Adv. Differential Equations, 8(12):1499-1532, 2003.

[35] Takéo Takahashi, Marius Tucsnak, and George Weiss. Stabilization of a fluid-rigid body system. J. Differential Equations, 259(11):6459-6493, 2015.

[36] Roger Temam. Problèmes mathématiques en plasticité, volume 12 of Méthodes Mathématiques de l'Informatique [Mathematical Methods of Information Science]. Gauthier-Villars, Montrouge, 1983. 